\title{
Kinetic Acidity of Aliphatic Hydrocarbons. Hydrogen Isotope Exchange with Cesium Cyclohexylamide in Cyclohexylamine
}

\author{
Andrew Streitwieser,* Tom A. Keevil, David R. Taylor and Edward C. \\ Dart \\ University of California, Department of Chemistry, Berkeley, CA 94720- \\ 1460
}

\section{Supporting Information}

\section{Contents}

Table S1. Run K3. Deuterodeprotonation of neopentane and hexamethylethane at 50.0 ${ }^{\circ} \mathrm{C}$.

Table S2. Deuterium analyses in Run K5 $\left(50.3{ }^{\circ} \mathrm{C}\right)$.

p. S2

Table S3. Run K6. Tritiodeprotonation in CHA-t

p. S3

Table S4. Run K7. Tritiodeprotonation in CHA-t

p. S3

Table S5. Run K8. Deuterodeprotonation in $\mathrm{CHA}-\mathrm{d}_{2}$ at $50.3^{\circ} \mathrm{C}$

p. S3

Table S6. Microwave Analysis of Deuteriated Propane and Isobutane from Run K8 p. S4

Table S7. Run DRT2 Tritiodeprotonation of cyclohexane and tetramethylbutane p. S4

Table S8. Run DRT5, Tritiodeprotonation of cyclohexane, neopentane and tetramethylbutane

p. S5

Table S9. Run DRT7. Deuteriodeprotonaion of methane, ethane and neopentane p. S5

Table S10 Run DRT8. Deuteriodepotonation of methane and cyclopropane p. S6

Table S11 Run DRT9. Tritiodeprotonation of cyclohexane and neopentane p. S6

$\begin{array}{ll}\text { Table S12 Run ECD6 p. S6 } & \text { p }\end{array}$

$\begin{array}{ll}\text { Table S13 Run ECD7 } & \text { p. S7 }\end{array}$

CsCHA SI v2.1.doc - S1 - 
Figure S1. Run DRT5: Tritiodeprotonation of NeoPeH and TMB p. S7

$\begin{array}{ll}\text { Figure } S 2 . \mathrm{Ln}(\% \mathrm{D}) \text { of nortrcyclene-3-d } & \text { p. S8 }\end{array}$

$\begin{array}{ll}\text { Figure S3. Neopentane compared to cyclohexane } & \text { p. S9 }\end{array}$

Figure S4. Run K3, deuterodeprotonation of neopentane and tetramethylbutane p. S10

Figure S5. Kinetic plots for ethane, propane and isobutane in run K8. p. S11

Figure S6. Deuterium incorporation into propane and isobutane vs ethane in run $\mathrm{K} 5$

Figure S7. Tritiodeprotonation of norbornane and adamantine p. S13

$\begin{array}{ll}\text { Chart S1 Abbreviations and acronyms used } & \text { p. S13 }\end{array}$

$\begin{array}{ll}\text { Chart S2 Spreadsheet codes for simulation of kinetics. } & \text { p. S14 }\end{array}$

Table S1. Run K3. Deuterodeprotonation of neopentane and hexamethylethane at 50.0 ${ }^{\circ} \mathrm{C}$.

\begin{tabular}{llcc} 
Point & Time $\left(10^{-6} \mathrm{~s}\right)$ & $\mathrm{NeoPeH}\left(\% \mathrm{~d}_{0}\right)$ & TMB $\left(\% \mathrm{~d}_{0}\right)$ \\
\hline 0 & 0.00 & 100.0 & 100.0 \\
1 & 0.09 & 98.4 & 99.0 \\
2 & 0.24 & 97.7 & 100.0 \\
3 & 0.49 & 97.3 & 99.9 \\
4 & 0.85 & 97.4 & 99.7 \\
5 & 1.45 & $\mathrm{a}$ & 99.4 \\
6 & 2.14 & 96.2 & 99.2 \\
7 & 3.00 & 95.2 & 98.7 \\
8 & 4.29 & 93.8 & 98.5 \\
9 & 7.08 & 92.1 & 97.1
\end{tabular}

(a). Sample accidentally lost before analysis.

Table S2. Deuterium analyses in Run K5 $\left(50.3^{\circ} \mathrm{C}\right)$.

\begin{tabular}{|c|c|c|c|}
\hline $\begin{array}{c}\text { Time } \\
10^{-6} \mathrm{sec}\end{array}$ & $\begin{array}{c}\text { Ethane } \\
\% \mathrm{~d}_{1}\end{array}$ & $\begin{array}{c}\text { Propane } \\
\% \mathrm{~d}_{1}\end{array}$ & $\begin{array}{c}\text { Isobutane } \\
\% \mathrm{~d}_{1}\end{array}$ \\
\hline 0.563 & 0.53 & 0.00 & 0.75 \\
1.178 & 1.22 & 1.90 & 1.83 \\
2.648 & 2.31 & & 2.90 \\
3.496 & 4.42 & 3.25 & 4.00 \\
5.581 & 5.10 & 6.02 & 6.40
\end{tabular}




\begin{tabular}{l|lll|}
8.329 & 6.00 & 7.21 & 7.41 \\
9.125 & 6.63 & 6.92 & 7.72 \\
\hline
\end{tabular}

Table S3. Run K6. Tritiodeprotonation in CHA-t containing 0.16M TMB, $0.24 \mathrm{M} \mathrm{CHX}, 0.15 \mathrm{M}$ p-xylene and $0.78 \mathrm{M}$ neoPeH at $50.3{ }^{\circ} \mathrm{C}$. GC response factors: area/mmole: CHX, $4.20 \times 10^{6}$; neoPeH, $7.12 \times 10^{6}$; TMB, $5.06 \times 10^{6}$.

\begin{tabular}{llll} 
Time, hr & \multicolumn{1}{c}{$\begin{array}{c}\mathrm{CHX} \\
10^{3} \\
\mathrm{dpm} / \mathrm{A}\end{array}$} & $\begin{array}{c}\mathrm{NeoPeH} \\
10^{3} \\
\mathrm{dpm} / \mathrm{A}\end{array}$ & $\begin{array}{c}\mathrm{TMB} \\
10^{3} \\
\mathrm{dpm} / \mathrm{A}\end{array}$ \\
\hline 142.8 & 3.90 & 18.12 & 12.15 \\
313.5 & 6.15 & 28.55 & 17.43 \\
743.1 & 13.56 & 64.22 & 48.51 \\
886.8 & 13.80 & 123.0 & 51.72 \\
1078.8 & 22.50 & 113.3 & 81.57 \\
1532.3 & 47.50 & 224.0 & 177.9 \\
2066.8 & 53.61 & 265.0 & 192.4 \\
2928.2 & 63.15 & 324.0 & 252.1 \\
infinitya & 59142 & 34887 & 73636
\end{tabular}

(a) Derived from p-xylene corrected for relative gc response.

Table S4. Run K7. Tritiodeprotonation in CHA-t (dpm/gc area) containing 0.19M norbornana (Nor) and $0.12 \mathrm{M}$ adamantane (Ada) at $50.3^{\circ} \mathrm{C}$. GC response factors: $\mathrm{CHX}$, 2.38E-7; Nor, 2.40E-7; Ada 1.47E-7 mmole/area.

\begin{tabular}{cccc} 
Time, $\mathrm{hr}$ & $\mathrm{CHX}$ & Nor & Ada \\
\hline 168.1 & 4.69 & 72.29 & 2.02 \\
338.6 & 7.46 & & 2.3 \\
778.2 & 10.86 & 106.8 & 2.88 \\
911.7 & 14.15 & 113.5 & 3.5 \\
1103 & 15.9 & 114.7 & 3.43 \\
1556 & 19.36 & 139.6 & 4.28 \\
1824 & 22.03 & 155.9 & 5.19 \\
2690 & 35.02 & 163 & \\
inf & 79396 & 80064 & 49039
\end{tabular}

Table S5. Run K8. Deuterodeprotonation in $\mathrm{CHA}-\mathrm{d}_{2}$ at $50.3{ }^{\circ} \mathrm{C}: \% \mathrm{~d}_{1}$ by mass spectral analysis. Amounts in solution are ethane, 79.2\%, propane, 92.8\%, isobutene, $96.1 \%$. 


\begin{tabular}{cccc} 
Time, hr & EtH & PrH & iBuH \\
\hline 114 & 0.57 & 1.37 & \\
667 & 2.66 & 1.92 & 1.24 \\
1650 & 10.21 & 9.37 & 8.07 \\
2826 & 19.22 & 18.17 & 16.41 \\
3714 & 24.9 & 22.86 & 20.34 \\
6425 & 25.22 & 23.23 & 21.4
\end{tabular}

Table S6. Microwave Analysis of Deuteriated Propane and Isobutane from Run K8 Compound Percent

$\begin{array}{ll}\mathrm{C}_{3} \mathrm{H}_{8} & 72.4 \pm 0.6^{\mathrm{a}} \\ \mathrm{CH}_{3} \mathrm{CH}_{2} \mathrm{CH}_{2} \mathrm{D} & 28.9^{\mathrm{b}}\left(23.9^{\mathrm{c}}\right) \\ \mathrm{CH}_{3} \mathrm{CH}_{2} \mathrm{CHD}_{2}+ & \\ \quad \mathrm{CH}_{2} \mathrm{DCH}_{2} \mathrm{CH}_{2} \mathrm{D} & 3.3^{\mathrm{c}} \\ \mathrm{CH}_{3} \mathrm{CHDCH}_{3} & <0.4^{\mathrm{d}} \\ \mathrm{C}_{4} \mathrm{H}_{10} & 71.8 \pm 0.8^{\mathrm{e}} \\ \left(\mathrm{CH}_{3}\right)_{2} \mathrm{CHCH}_{2} \mathrm{D} & 24.2^{\mathrm{c}} \\ \left(\mathrm{CH}_{3}\right)_{2} \mathrm{CHCHD}_{2}+ & \\ \left(\mathrm{CH}_{2} \mathrm{D}\right)_{2} \mathrm{CHCH}_{3} & 3.6^{\mathrm{c}} \\ \left(\mathrm{CH}_{3}\right)_{3} \mathrm{CD} & <0.8^{\mathrm{d}}\end{array}$

a. Intensity of $\mathrm{J}=60,6 Æ 61,5$ line compared to pure $\mathrm{C}_{3} \mathrm{H}_{8}$.

b. Nominal value for direct measurement. No correction for Stark coefficients.

c. Calculated from $\% \mathrm{~d}_{\mathrm{o}}$, assuming all deuterium in the primary position, and no isotope effects.

d. Upper limit; no signal detected.

e. Intensity of $\mathrm{J}=1$ Æ 2 line compared to pure $\mathrm{C}_{4} \mathrm{H}_{10}$

Table S7. Run DRT2 Tritiodeprotonation of cyclohexane and tetramethylbutane at $50{ }^{\circ} \mathrm{C}$ by proportional flow counting. 


\begin{tabular}{|c|c|c|c|c|c|c|}
\hline & \multicolumn{3}{|c|}{ TMB } & \multicolumn{3}{c|}{ CHX } \\
\hline Point no. & Area,A & Counts,C & $10^{3} \mathrm{C} / \mathrm{A}$ & Area,A & Counts,C & $10^{3} \mathrm{C} / \mathrm{A}$ \\
\hline 10 & 46180 & 2082 & 45.1 & 79882 & 430 & 5.4 \\
\hline 11 & 43789 & 2705 & 61.8 & 89551 & 789 & 8.8 \\
\hline 12 & 30393 & 2284 & 75.2 & 69641 & 730 & 10.5 \\
\hline
\end{tabular}

$\mathrm{GC}$ response: $\mathrm{CHX} / \mathrm{TMB}=1.124 \pm 0.005$.

Table S8. Run DRT5, Tritiodeprotonation of cyclohexane, neopentane and tetramethylbutane at $100^{\circ} \mathrm{C}$ by liquid scintillation counting.

\begin{tabular}{|c|c|c|}
\hline NeoPeH, dmp/area & TMB, dmp/area & CHX, dmp/area \\
\hline 0.0347 & 0.0316 & 0.0036 \\
\hline 0.0598 & 0.0450 & 0.0092 \\
\hline 0.0774 & 0.0662 & 0.0176 \\
\hline 0.148 & 0.1074 & 0.0312 \\
\hline 0.2338 & 0.1604 & 0.0474 \\
\hline 0.275 & 0.201 & 0.0479 \\
\hline 0.2902 & 0.2199 & 0.0573 \\
\hline
\end{tabular}

GC Response: $\mathrm{CHX} / \mathrm{NeoPeH}=1.46 ; \mathrm{CHX} / \mathrm{TMB}=1.21$

Table S9. Run DRT7. Deuteriodeprotonaion of methane, ethane and neopentane in CHA-d $\mathrm{d}_{2}, 0.0156 \mathrm{M} \mathrm{CsCHA}$, at $50^{\circ} \mathrm{C}$. Ethane was $0.157 \mathrm{M}$ with 0.735 dissolved; neopentane was $0.603 \mathrm{M}$ with 0.981 dissolved. The methane results were given previously. ${ }^{1}$

\begin{tabular}{|c|c|c|}
\hline Time, $10^{5} \mathrm{~s}$ & $\% \mathrm{C}_{2} \mathrm{H}_{6}$ & $\% \mathrm{C}_{5} \mathrm{H}_{12}$ \\
\hline 0 & 100 & 100 \\
\hline 6.04 & 96.1 & - \\
\hline 6.41 & 96.6 & 97.6 \\
\hline 7.07 & 96.0 & 97.4 \\
\hline 7.86 & 95.9 & 97.5 \\
\hline 12.57 & 94.3 & 96.2 \\
\hline 18.23 & 92.9 & 95.8 \\
\hline 25.38 & 91.6 & 94.6 \\
\hline 31.22 & 89.7 & 93.8 \\
\hline
\end{tabular}




\begin{tabular}{|l|l|l|}
\hline 38.22 & 89.6 & 93.2 \\
\hline 43.16 & 89.1 & 92.5 \\
\hline 49.44 & 85.3 & 92.2 \\
\hline
\end{tabular}

Table S10 Run DRT8. Deuteriodepotonation of 10.1 mmole methane and 35.0 mmole cyclopropane (0.965 in solution) in 663 mmole CHA- $\mathrm{d}_{2}$ 0.029M in CsCHA at 50 ${ }^{\circ} \mathrm{C}$.

\begin{tabular}{|c|c|c|c|c|c|c|c|c|c|}
\hline $\begin{array}{l}\text { time, } \\
10^{4} \mathrm{~s}\end{array}$ & $\begin{array}{l}\% \\
\mathrm{CH}_{4}\end{array}$ & $\begin{array}{l}\% \\
\mathrm{CH}_{3} \mathrm{D} \\
\end{array}$ & $\begin{array}{l}\% \\
\mathrm{C}_{3} \mathrm{H}_{8} \\
\end{array}$ & $\begin{array}{l}\% \\
\mathrm{C}_{3} \mathrm{H}_{5} \mathrm{D}\end{array}$ & $\begin{array}{l}\% \\
\mathrm{C}_{3} \mathrm{H}_{4} \mathrm{D}_{2}\end{array}$ & $\begin{array}{l}\% \\
\mathrm{C}_{3} \mathrm{H}_{3} \mathrm{D}_{3}\end{array}$ & $\begin{array}{l}\% \\
\mathrm{C}_{3} \mathrm{H}_{2} \mathrm{D}_{4}\end{array}$ & $\begin{array}{l}\% \\
\mathrm{C}_{3} \mathrm{HD}_{5}\end{array}$ & $\begin{array}{l}\% \\
\mathrm{C}_{3} \mathrm{D}_{8}\end{array}$ \\
\hline 0.097 & 99.5 & 0.5 & 50.5 & 35.3 & 12.2 & 2.0 & & & \\
\hline 0.166 & 99.0 & 1.0 & 18.2 & 35.7 & 30.3 & 13.0 & 2.8 & & \\
\hline 0.293 & 97.3 & 2.7 & 7.2 & 20.5 & 32.5 & 26.2 & 11.3 & 2.3 & \\
\hline 0.426 & 97.1 & 2.9 & 4.2 & 12.9 & 26.8 & 31.0 & 19.0 & 5.6 & 0.7 \\
\hline
\end{tabular}

Table S11 Run DRT9. Tritiodeprotonation of cyclohexane and neopentane at 50 ${ }^{\circ} \mathrm{C}$ by proportional flow counting.

\begin{tabular}{|c|c|c|c|c|c|c|}
\hline & \multicolumn{3}{|c|}{ NeoPeH } & \multicolumn{3}{c|}{ CHX } \\
\hline Point no. & Area,A & Counts,C & $10^{3} \mathrm{C} / \mathrm{A}$ & Area,A & Counts,C & $10^{3} \mathrm{C} / \mathrm{A}$ \\
\hline 1 & 12565 & 44 & 3.5 & 30726 & 14 & 0.46 \\
\hline 2 & 27060 & 505 & 18.4 & 67000 & 98 & 1.46 \\
\hline 3 & 87000 & 2119 & 24.4 & 279000 & 462 & 1.66 \\
\hline 4 & 72150 & 1923 & 26.7 & 101290 & 637 & 3.15 \\
\hline 5 & 45114 & 1426 & 31.6 & 93254 & 288 & 3.10 \\
\hline
\end{tabular}

$\mathrm{GC}$ response: $\mathrm{CHX} / \mathrm{NeoPeH}=0.889 \pm 0.005$.

Table S12 Run ECD6. Protodedeuteraton of norbornane-1-d; tritiodetprotonation of cyclohexane and tetramethylbutane at $101^{\circ} \mathrm{C}$.

\begin{tabular}{|c|c|c|}
\hline $\begin{array}{c}\mathrm{CHX}^{\mathrm{a}} \\
\mathrm{dpm} / \mathrm{mmole} \times 10^{5}\end{array}$ & $\begin{array}{c}\mathrm{TMB}^{\mathrm{a}} \\
\mathrm{dpm} / \mathrm{mmole} \times 10^{5}\end{array}$ & $\begin{array}{c}\text { Norbornane-1-d } \\
\% \mathrm{~d}\end{array}$ \\
\hline 0.714 & 0.09 & 76.1 \\
\hline 3.75 & 8.02 & \\
\hline 5,30 & 13.69 & \\
\hline 8.33 & 25.39 & \\
\hline 4.42 & 12.24 & \\
\hline 9.55 & 25.80 & 73.9 \\
\hline 6.00 & 18.75 & 70.4 \\
\hline 18.15 & 37.20 & \\
\hline 40.60 & 123.00 & \\
\hline
\end{tabular}




\section{\begin{tabular}{r|r}
55.70 & 145.5
\end{tabular}}

(a) infinity value (from p-xylene) is $587 \times 10^{5} \mathrm{dpm} / \mathrm{mmole} / \mathrm{H}$

Table S13 Run ECD7. Protodedeuteraton of nortricyclene-3-d; tritiodetprotonation of cyclohexane at $101^{\circ} \mathrm{C}$.

\begin{tabular}{|c|c|c|}
\hline Time, $\mathrm{m}$ & $\begin{array}{c}\mathrm{CHX} \\
\mathrm{dpm} / \mathrm{mmole} \times 10^{5}\end{array}$ & $\begin{array}{c}\text { Nortricyclene-3-d } \\
\% \mathrm{~d}\end{array}$ \\
\hline 30 & 1.63 & 91.25 \\
\hline 169 & 3.12 & 90.80 \\
\hline 539 & 5.20 & 89.4 \\
\hline 1510 & 7.23 & 87.8 \\
\hline 3255 & 8.16 & 88.0 \\
\hline 4358 & 7.80 & 87.25 \\
\hline 5534 & 10.10 & 86.5 \\
\hline 11626 & 9.60 & 87.0 \\
\hline 24480 & 14.20 & 85.4 \\
\hline
\end{tabular}

(a) infinity value is $742 \times 10^{5} \mathrm{dpm} / \mathrm{mmole} / \mathrm{H}$

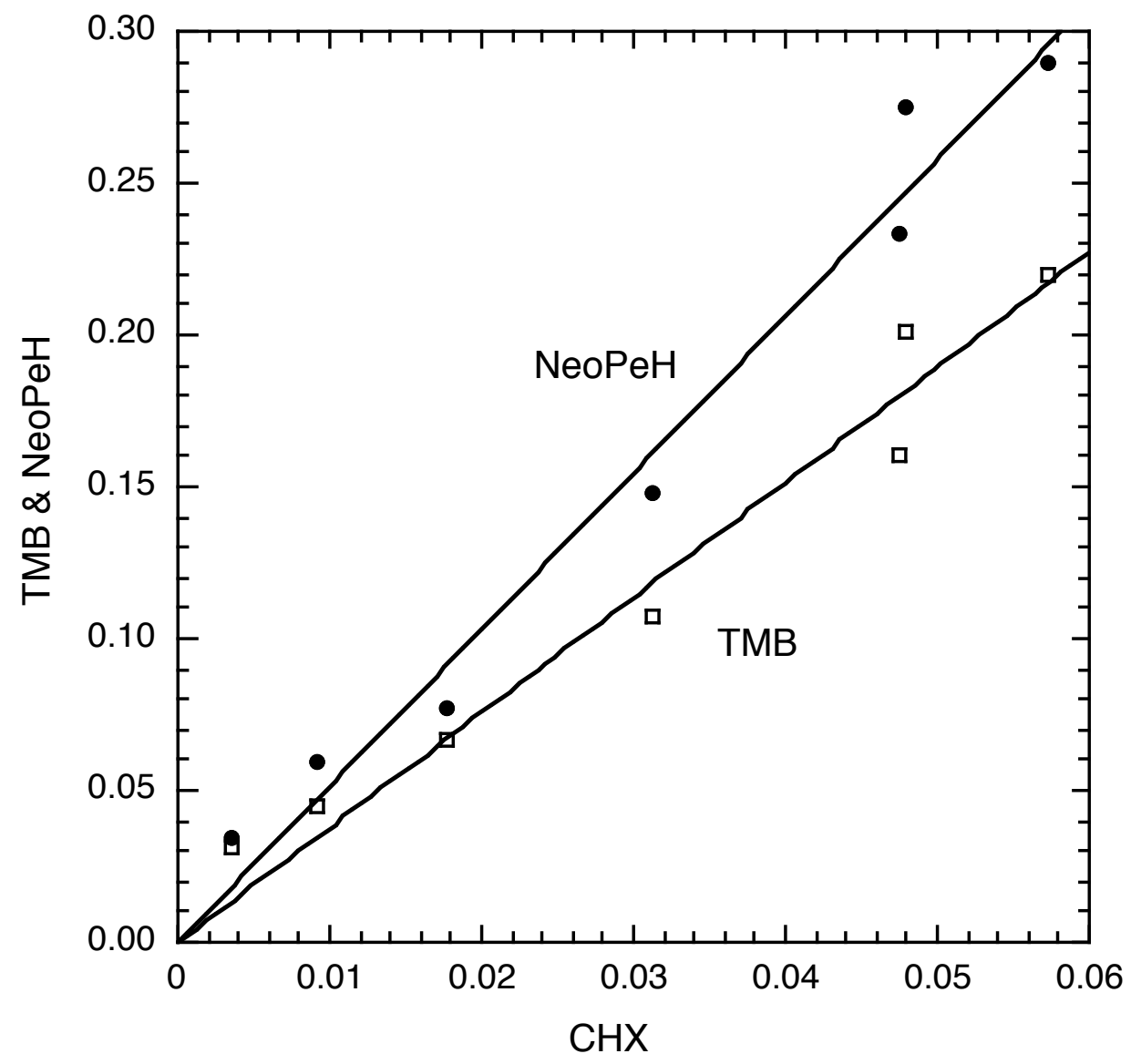


Figure S1. Run DRT5: Tritiodeprotonation of NeoPeH (filled circles) and TMB (squares) vs CHX. Slopes shown are $5.16 \pm 0.17\left(\mathrm{R}^{2}=0.975\right) ; 3.79 \pm 0.15\left(\mathrm{R}^{2}=0.963\right)$.

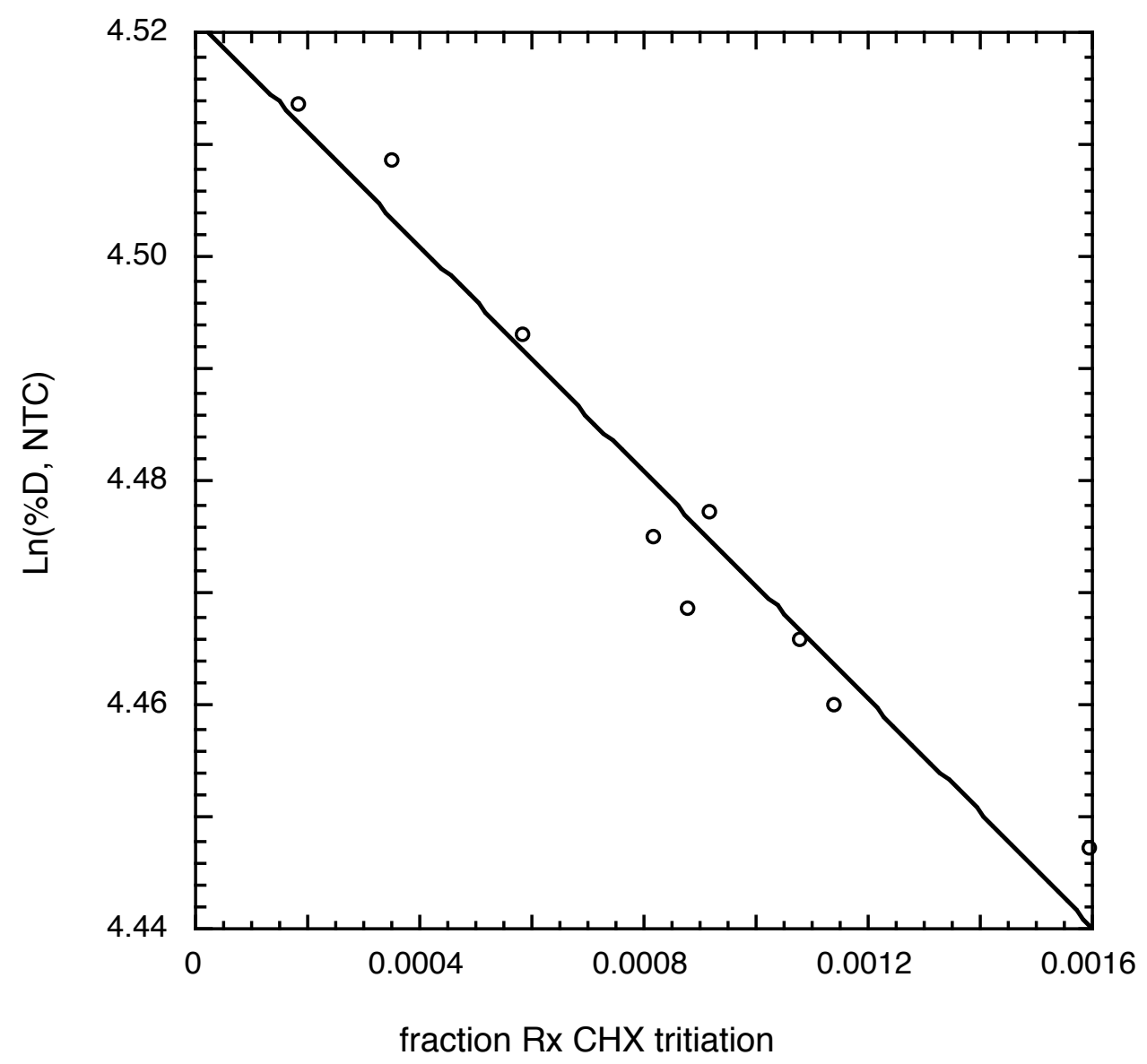

Figure S2. Ln(\%D) of nortrcyclene-3-d plotted against fraction of tritiodeprotonation reaction of cyclohexane $(\mathrm{dpm} / \mathrm{mmole} / \mathrm{infinity})$ at $101{ }^{\circ} \mathrm{C}$. The line shown is $4.521 \pm 0.004$ $-(50.6 \pm 4.3) x ; R^{2}=0.952$. 


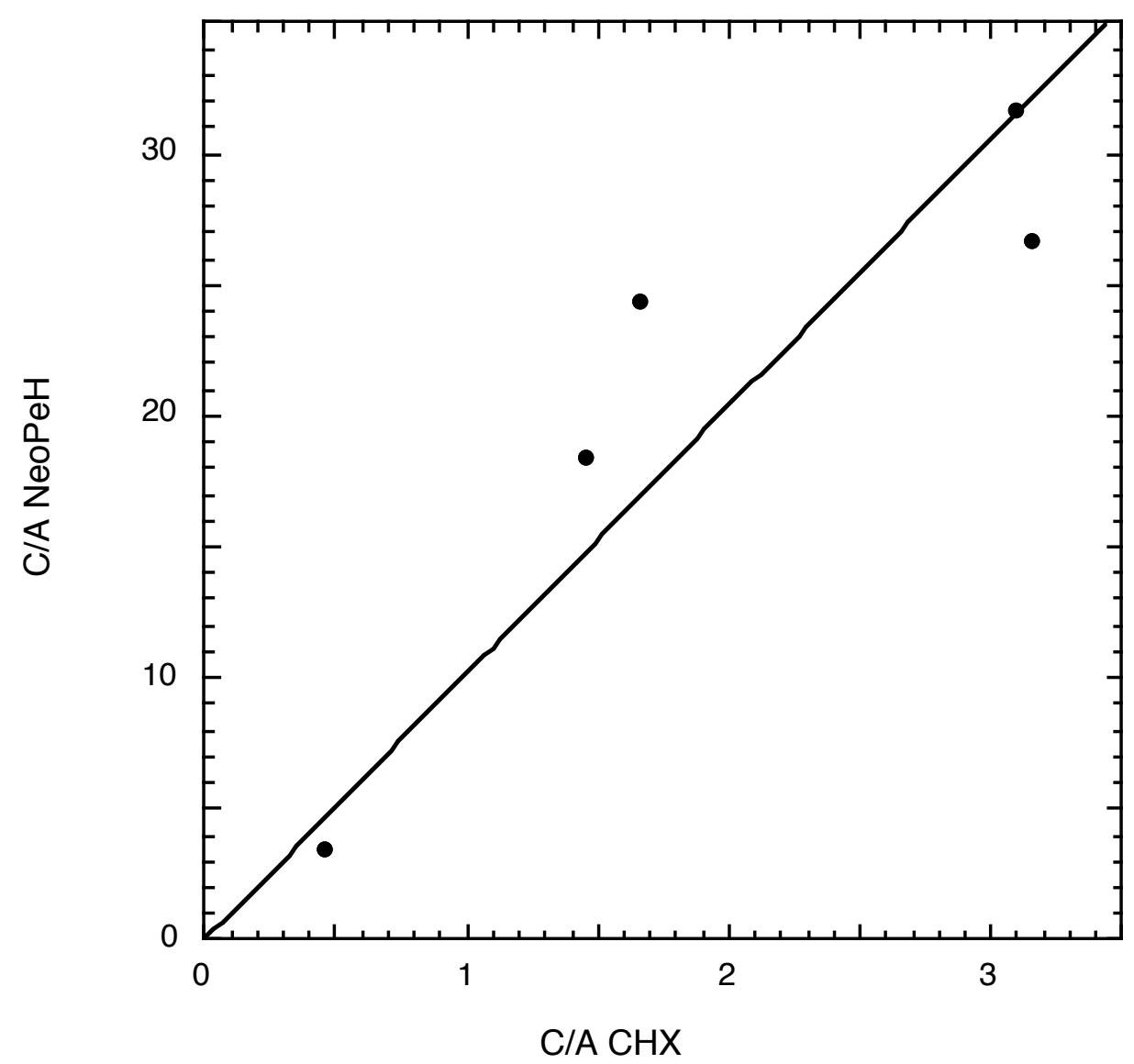

Figure S3. Counts/gc area $\left(\mathrm{x} 10^{-3}\right)$ for neopentane compared to cyclohexane in CsCHA/CHA-t at $50^{\circ} \mathrm{C}$ from run DRT9. Slope of line is 10.,2 \pm 1.0 . 


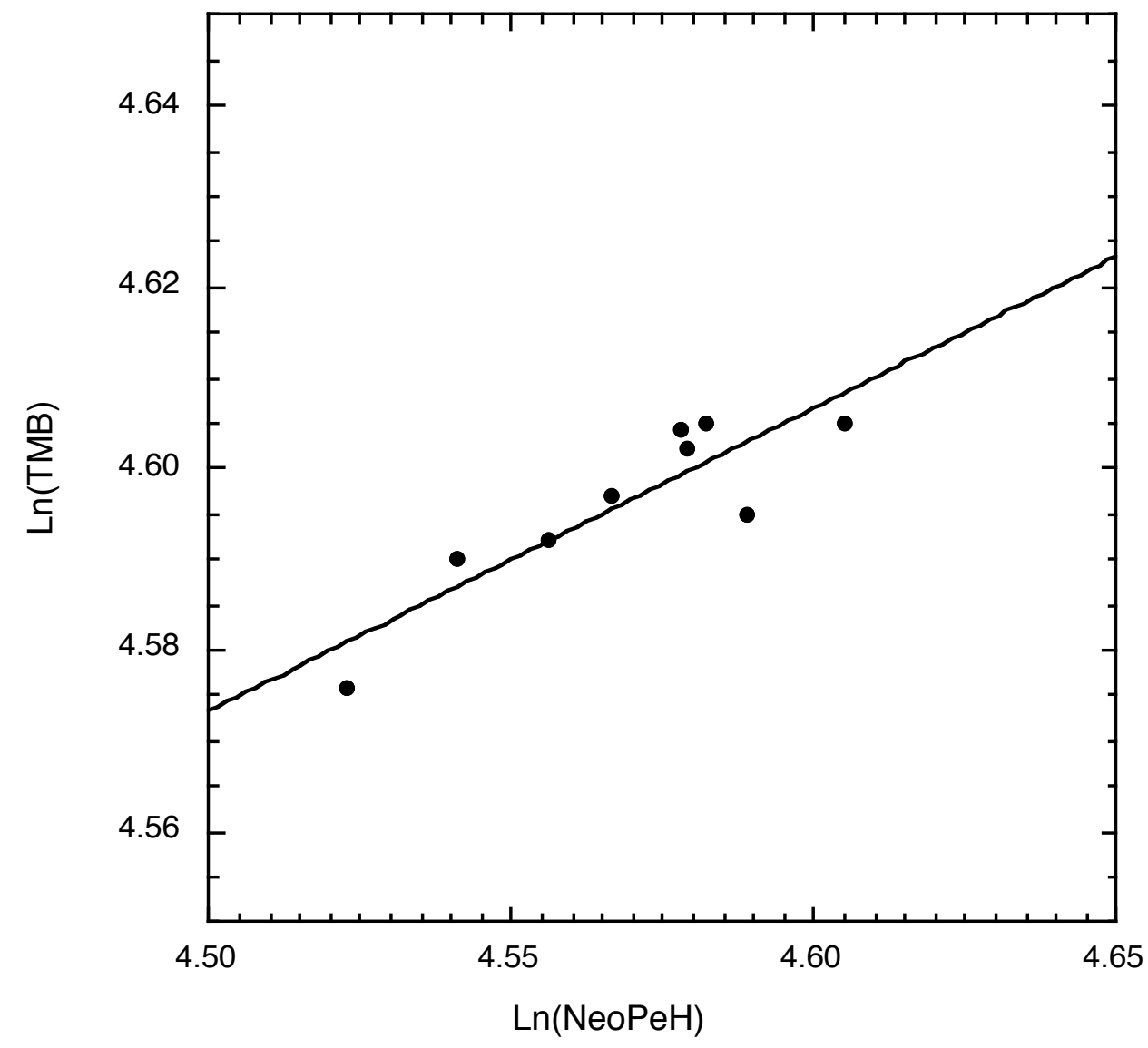

Figure S4. Run K3, deuterodeprotonation of neopentane and tetramethylbutane with CsCHA in CHA- $\mathrm{d}_{2}$. The line shown is $3.07 \pm 0.31+$ $(0.334 \pm 0.067) x ; R^{2}=0.779$. 


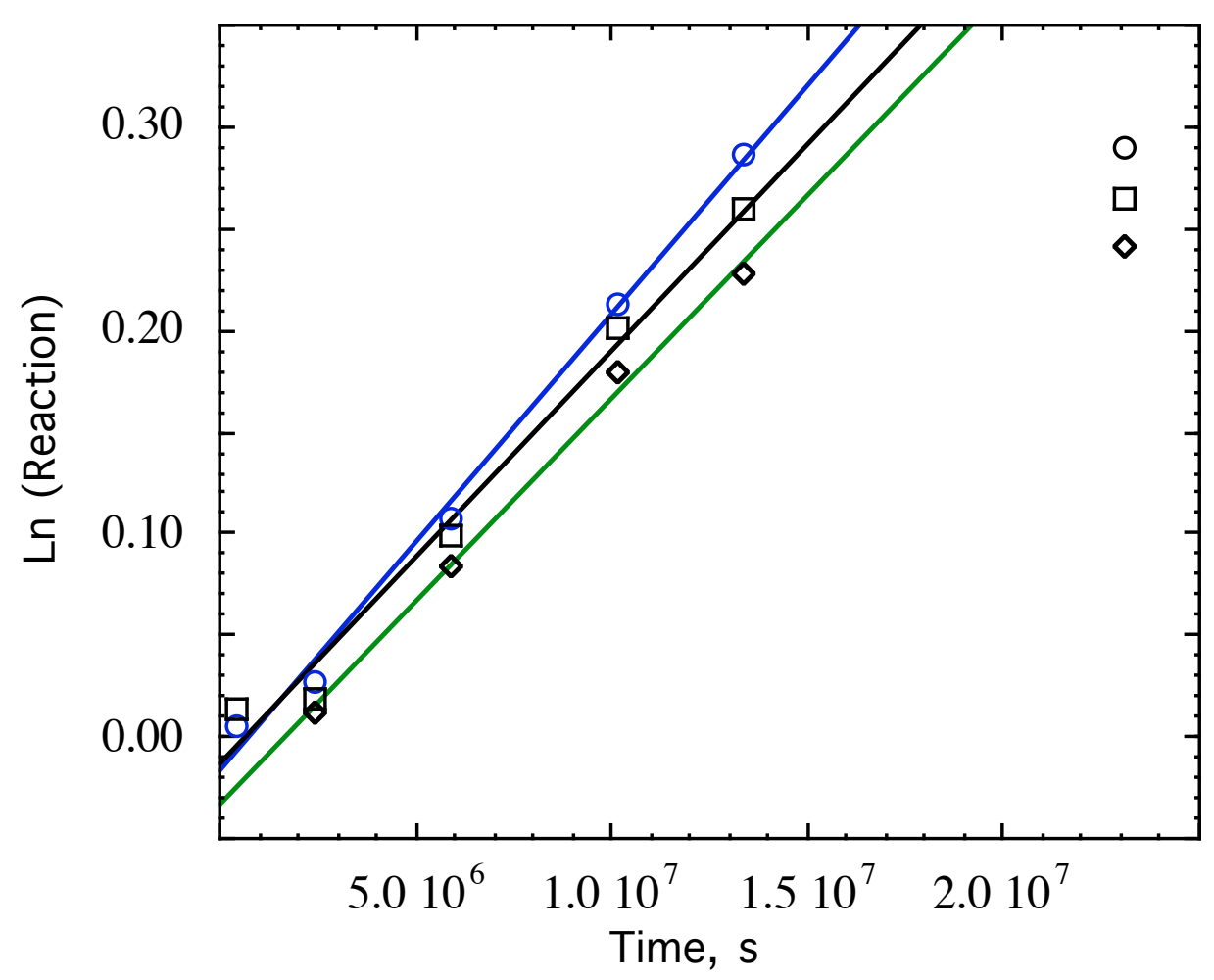

Figure S5. Pseudo-first order kinetic plots for ethane (circles), propane (squares) and isobutane (diamonds) in run $\mathrm{K} 8$. The outlying points are not included in the least squares lines that have the equations (respectively), $-0.017+(2.239 \pm 0.10) \times 10^{-8} \mathrm{x}\left(\mathrm{R}^{2}=0.994\right)$; $-0.012+(2.024 \pm 0.15) \times 10^{-8} \mathrm{x}, \mathrm{R}^{2}=0.984 ;-0.033+(1.999 \pm 0.10) \times 10^{-8} \mathrm{x} ; \mathrm{R}^{2}=0.995$. 


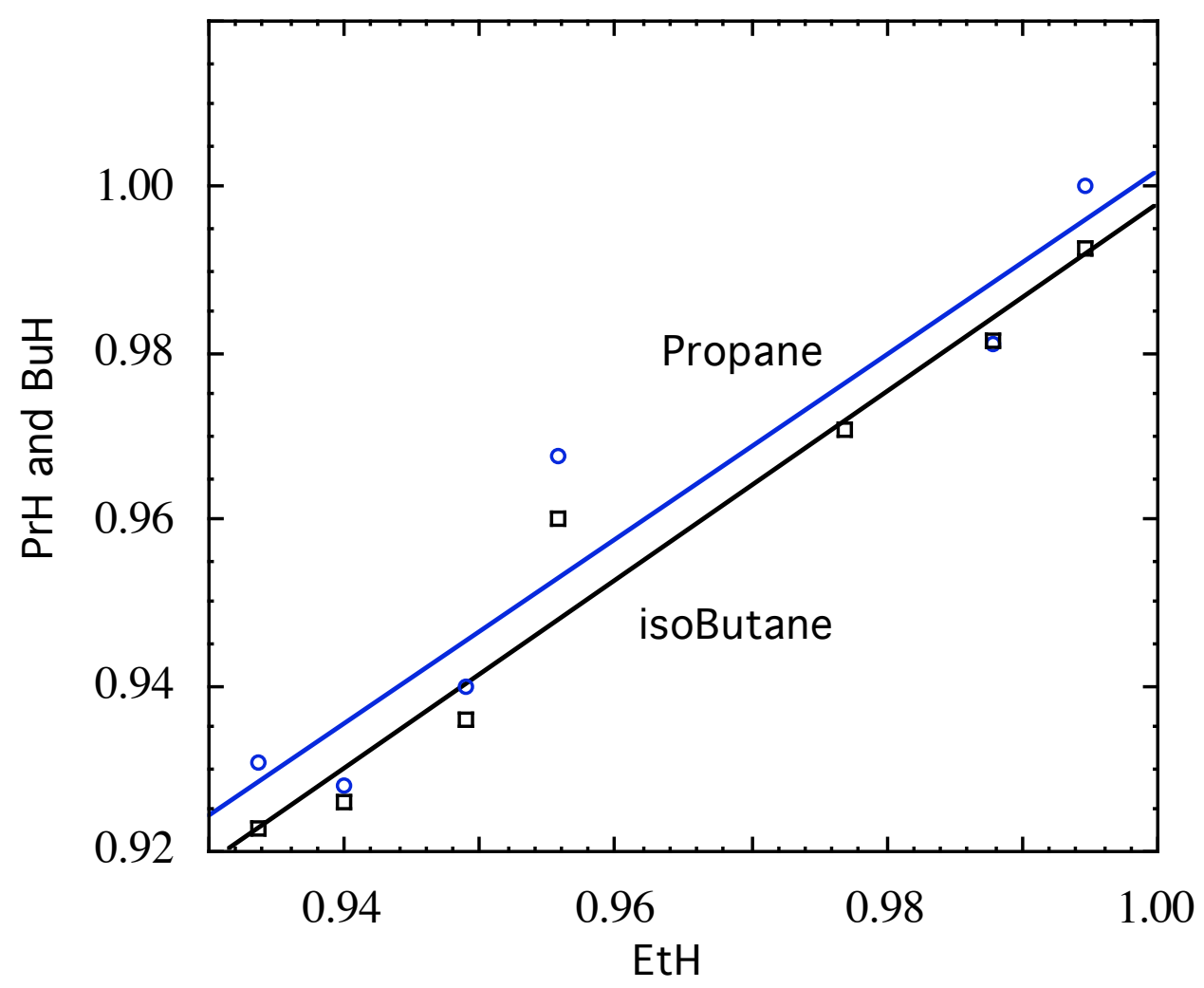

Figure S6. Deuterium incorporation into propane and isobutane vs ethane in Run K5. The equations of the lines shown are: propane, $-0.112+(1.11 \pm 0.17) \mathrm{x}, \mathrm{R}^{2}=0.914$; isobutane, $-0.138+(1.14 \pm 0.10) \mathrm{x}, \mathrm{R}^{2}=0.960$. 


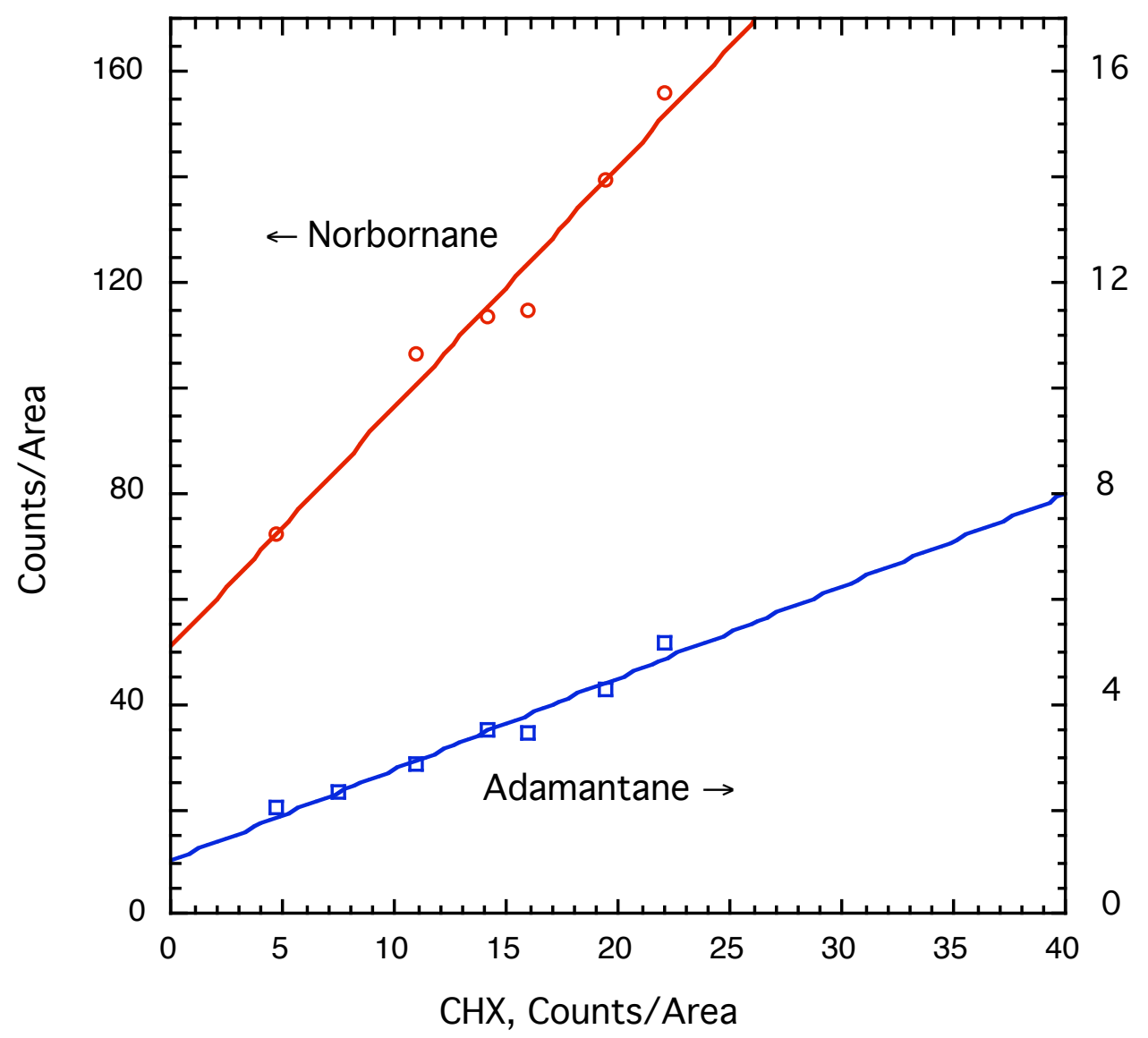

Figure S7. Tritiodeprotonation of norbornane (circles) and adamantane (squares) compared to cyclohexane. Lines shown are 50.9 $\pm 6.7+(4.57 \pm 0.43) \times\left(R^{2}=0.966\right)$ and $1.03 \pm 0.02+(0.174 \pm 0.016) \times\left(\mathrm{R}^{2}=0.961\right)$.

Chart S1 Abbreviations and acronyms used:

CHA cyclohexylamine

CHA-d cyclohexylamine- $\mathrm{N}-d_{2}$

CHA-t cyclohexylamine-N- $t$

LiCHA lithium cyclohexylamide

CsCHA cesium cyclohexylamide

EtH ethane

$\mathrm{PrH} \quad$ propane

$\mathrm{iBuH}$ isobutane

neoPeH neopentane

TMB 2,2,3,3-tetramethylbutane

$\mathrm{MeH}$ methane

CHX cyclohexane

Nor norbornane

Ada adamantane 
cPrH cyclopropane

NTC nortricyclene

\section{Chart S2 Spreadsheet codes for simulation of kinetics.}

Each entry for $D_{i}$ starts with the previous $D_{i}$ and adds an increment for a $\Delta t=T$. $\mathrm{L}=$ primary isotope effect, $\mathrm{k}_{\mathrm{H}} / \mathrm{k}_{\mathrm{D}}$ (taken as 4$\left.) ; \mathrm{Mu}=\left(\mathrm{L}^{*} \mathrm{n}_{\mathrm{H}} / \mathrm{n}_{\mathrm{D}}\right) /\left(\left(\mathrm{L}^{*} \mathrm{n}_{\mathrm{H}} / \mathrm{n}_{\mathrm{D}}\right)+1\right)\right)$ in which $\mathrm{n}_{\mathrm{H}}=$ mole fraction of available hydrogens in the solvent; $\mathrm{d}=$ secondary isotope effect for methane, $\mathrm{k}_{\mathrm{H}} / \mathrm{k}_{\mathrm{D}}$, and $\mathrm{e}=$ secondary isotope effect for cyclopropane, $\mathrm{k}_{\mathrm{H}} / \mathrm{k}_{\mathrm{D}}$ (both were taken as 1.1).

Methane:

D1: $\quad=\mathrm{D} 1+(-(3 * \mathrm{D} 1 / \mathrm{d})-(\mathrm{D} 1 / \mathrm{L})+(1-\mathrm{Mu}) *(4 * \mathrm{D} 0+(\mathrm{D} 1 / \mathrm{L}))+$ $\left.\mathrm{Mu}^{*}((3 / \mathrm{d}) * \mathrm{D} 1+(2 / \mathrm{L}) *(\mathrm{D} 2 / \mathrm{d}))\right) * \mathrm{~T} * \mathrm{k}$

$\mathrm{D} 2: \quad=\mathrm{D} 2+(-(2 * \mathrm{D} 2 / \mathrm{d})-(2 * \mathrm{D} 2 / \mathrm{L})+(1-\mathrm{Mu}) *(3 * \mathrm{D} 1+(2 * \mathrm{D} 2 / \mathrm{L}))+$ $\left.\mathrm{Mu}^{*}((2 / \mathrm{d}) * \mathrm{D} 2+(3 / \mathrm{L}) *(\mathrm{D} 3 / \mathrm{d}))\right) *(\mathrm{~T} * \mathrm{k} / \mathrm{d})$

D3: $\quad=\mathrm{D} 3+(-(\mathrm{D} 3 / \mathrm{d})-(3 * \mathrm{D} 3 / \mathrm{L})+(1-\mathrm{Mu}) *(2 * \mathrm{D} 2+(3 / \mathrm{L}) * \mathrm{D} 3)+\mathrm{Mu} *((\mathrm{D} 3 / \mathrm{d})+$ $(4 / \mathrm{L}) *(\mathrm{D} 4 / \mathrm{d})))^{*}(\mathrm{~T} / \mathrm{d}) *(\mathrm{k} / \mathrm{d})$

D4: $\quad=\mathrm{D} 4+((1-\mathrm{Mu}) * \mathrm{D} 3-(\mathrm{Mu} / \mathrm{L}) * 4 * \mathrm{D} 4) *(\mathrm{k} / \mathrm{d}) *(\mathrm{~T} / \mathrm{d}) / \mathrm{d}$

Cyclopropane:

$$
\begin{aligned}
& \text { D0: } \quad=\mathrm{D} 0+\mathrm{k}^{*}((\mathrm{Mu} * \mathrm{D} 1 / \mathrm{L})-6 * \mathrm{D} 0 *(1-\mathrm{Mu}))^{* \mathrm{~T}} \\
& \mathrm{D} 1: \quad=\mathrm{D} 1+\left((5 / \mathrm{e}) * \mathrm{D} 1 *(\mathrm{Mu}-1)-(\mathrm{D} 1 / \mathrm{L})+(1-\mathrm{Mu})^{*}(6 * \mathrm{D} 0+(\mathrm{D} 1 / \mathrm{L}))+\right. \\
& (\mathrm{Mu} / \mathrm{e}) * 2 *(\mathrm{D} 2 / \mathrm{L}))^{*} \mathrm{k}^{* \mathrm{~T}} \\
& \mathrm{D} 2: \quad=\mathrm{D} 2+(((-4) * \mathrm{D} 2 / \mathrm{e})-(2 * \mathrm{D} 2 / \mathrm{L})+(1-\mathrm{Mu}) *(5 * \mathrm{D} 1+(2 * \mathrm{D} 2 / \mathrm{L}))+ \\
& \left.\mathrm{Mu}\left(\left(3^{*} \mathrm{D} 3 / \mathrm{L} * \mathrm{e}\right)+(4 * \mathrm{D} 2 / \mathrm{e})\right)\right) *(\mathrm{k} / \mathrm{e}) *(\mathrm{~T}) \\
& \mathrm{D} 3: \quad=\mathrm{D} 3+((-3 * \mathrm{D} 3 / \mathrm{e})-(3 * \mathrm{D} 3 / \mathrm{L})+(1-\mathrm{Mu}) *((3 * \mathrm{D} 3 / \mathrm{L})+4 * \mathrm{D} 2)+ \\
& \mathrm{Mu} *(3 *(\mathrm{D} 3 / \mathrm{e})+(4 * \mathrm{D} 4 /(\mathrm{L} * \mathrm{e})))) *(\mathrm{k} / \mathrm{e}) *(\mathrm{~T} / \mathrm{e}) \\
& \mathrm{D} 4: \quad=\mathrm{D} 4+((-2 * \mathrm{D} 4 / \mathrm{e})-(4 * \mathrm{D} 4 / \mathrm{L})+(1-\mathrm{Mu}) *((4 * \mathrm{D} 4 / \mathrm{L})+3 * \mathrm{D} 3)+ \\
& \mathrm{Mu} *(2 *(\mathrm{D} 4 / \mathrm{e})+(5 * \mathrm{D} 5 /(\mathrm{L} * \mathrm{e})))) *(\mathrm{k} / \mathrm{e}) *(\mathrm{~T} / \mathrm{e}) / \mathrm{e} \\
& \mathrm{D} 5: \quad=\mathrm{D} 5+((-\mathrm{D} 5 / \mathrm{e})-(5 * \mathrm{D} 5 / \mathrm{L})+(1-\mathrm{Mu}) *((5 * \mathrm{D} 5 / \mathrm{L})+(2 * \mathrm{D} 4))+ \\
& \mathrm{Mu} *((\mathrm{D} 5 / \mathrm{e})+(6 * \mathrm{D} 6 / \mathrm{L} * \mathrm{e}))) *(\mathrm{k} / \mathrm{e}) *(\mathrm{~T} / \mathrm{e}) /(\mathrm{e} * \mathrm{e}) \\
& \mathrm{D} 6: \quad=\mathrm{D} 6+(((1-\mathrm{Mu}) * \mathrm{D} 5-(\mathrm{Mu} 6 * \mathrm{D} 6 / \mathrm{L})) *(\mathrm{k} / \mathrm{e}) *(\mathrm{~T} / \mathrm{e})) /\left(\mathrm{e}^{*} \mathrm{e}^{*} \mathrm{e}\right)
\end{aligned}
$$

\section{Reference}

(1) Streitwieser, A.; Taylor, D. R. Arkivok 2002, 70-78. 